\title{
TUBERCULOSIS EN TRABAJADORES DE SALUD EN EL PERÚ, 2013-2015
}

\author{
Mirtha Gabriela Soto-Cabezas ${ }^{1, a}$, Ana María Chávez-Pachas ${ }^{1, b}$, Juan Carlos Arrasco-Alegre ${ }^{1, a}$, \\ Martin Javier Alfredo Yagui-Moscoso ${ }^{1, a}$
}

\begin{abstract}
RESUMEN
Objetivos. Describir las características epidemiológicas de la tuberculosis en trabajadores de salud del Perú. Materiales y métodos. Se realizó un estudio descriptivo que incluyó a todos los trabajadores de salud con tuberculosis notificados al sistema nacional de vigilancia epidemiológica del Ministerio de Salud (MINSA) del Perú, durante los años 2013, 2014 y 2015. Resultados. Se notificaron 755 casos de tuberculosis en trabajadores de salud, el $60 \%$ laboraban en hospitales, $28 \%$ en establecimientos del primer nivel de atención y $12 \%$ en establecimientos privados. $57 \%$ de los casos laboraban en establecimientos de salud de Lima Metropolitana y el Callao. La edad promedio de los trabajadores fue de 38 años (rango 19 a 89 años) y 63,6\% fueron mujeres; $6,1 \%$ de los casos fueron resistentes, principalmente tuberculosis multidrogorresistente; $67 \%$ de los casos fueron tuberculosis pulmonar con confirmación bacteriológica. Profesionales y técnicos de la salud representaron el $82,5 \%$ de los casos, consultorios, hospitalización y emergencia, fueron las áreas donde laboraban el $55,2 \%$ de los casos. Conclusiones. La tuberculosis constituye un riesgo laboral importante para los trabajadores de salud de los establecimientos de salud públicos y privados, afecta principalmente al grupo de profesionales y técnicos de la salud que realizan labores en áreas de cuidado y atención directa con pacientes en grandes hospitales de Perú.
\end{abstract}

Palabras clave: Tuberculosis; Personal de Salud; Vigilancia epidemiológica (fuente: DeCS BIREME).

\section{TUBERCULOSIS IN HEALTH WORKERS IN PERU, 2013-2015}

\begin{abstract}
Objectives. To describe the characteristics of tuberculosis epidemiology in Peruvian health workers. Materials and methods. A descriptive study was performed. It included all health workers with tuberculosis listed in the national epidemiological surveillance system of the Peruvian Ministry of Health, during 2013, 2014, and 2015. Results. A total of 755 cases of tuberculosis in health workers were reported: $60 \%$ worked in hospitals, $28 \%$ worked in primary-care facilities, and $12 \%$ worked in private facilities. In $57 \%$ of the cases, they worked in health facilities in Lima Metropolitan area and Callao. The average age of workers was 38 years (ranging from 19 to 89 years), and $63.6 \%$ were women. Of the cases, $6.1 \%$ were resistant, mainly multidrug-resistant tuberculosis, $67 \%$ of the cases had bacteriological confirmation of pulmonary tuberculosis. Health professionals and technicians represented $82.5 \%$ of the cases, and $55.2 \%$ corresponded to doctor's offices, hospital admissions, and emergency rooms, the areas where they worked. Conclusions. Tuberculosis poses a significant work risk for health workers of both public and private facilities. It mainly affects health professionals and technicians providing direct care and assistance to patients in large Peruvian hospitals.
\end{abstract}

Key words: Tuberculosis; Health Personnel; Epidemiological Surveillance (source: MeSH NLM)

\section{INTRODUCCIÓN}

La tuberculosis (TB) es un problema de salud mundial que afecta predominantemente a la población económicamente activa y es considerada una enfermedad ocupacional (1). La transmisión de la TB es un riesgo laboral en los establecimientos de salud, más en aquellos donde se atienden grandes colectivos de pacientes. Los pacientes bacilíferos no diagnosticados, y los que no reciben tratamiento adecuado, constituyen el mayor riesgo de contagio para los trabajadores de salud (TS) ${ }^{(2)}$.

Varios estudios han mostrado un alto riesgo de infección tuberculosa latente en TS en países de medianos y bajos ingresos, así como en aquellos con mayor prevalencia de TB en población general ${ }^{(2-5)}$, donde el riesgo de desarrollar una infección tuberculosa latente o enfermedad activa por TB fue mayor en trabajadores de salud que en la población general y, el riesgo existe

\footnotetext{
Centro Nacional de Epidemiología, Prevención y Control de Enfermedades, Ministerio de Salud. Lima, Perú.

Facultad de Medicina, Universidad Nacional San Luis Gonzaga. Ica, Perú.

a Médico cirujano; ${ }^{b}$ Licenciada en enfermería

Recibido: 17/05/2016 Aprobado: 12/10/2016
} 
aun en situaciones de baja prevalencia de TB en la población ${ }^{(2)}$. La ocurrencia de brotes nosocomiales ha sido un tema reportado por varios autores en diferentes países, los mismos que afectaron tanto a TS como a pacientes, algunos causados por formas de TB resistente ${ }^{(6-10)}$. Unos cuantos estudios investigaron las características de los TS con enfermedad activa y estimaron la incidencia de enfermedad tuberculosa en este grupo ocupacional, evidenciando una incidencia de enfermedad mayor al de la población general y 3 a 6 veces mayor riesgo de desarrollar la enfermedad que la población en general ${ }^{(11-13)}$.

En el Perú, la tuberculosis es una enfermedad endémica, con altas tasas de incidencia y trasmisión activa en varios departamentos, según el último informe de la Organización Panamericana de la Salud, Perú es el segundo país con la más alta carga de TB en la región de América Latina y el Caribe ${ }^{(14)}$, para el año 2014 se estimó una incidencia anual de 90 casos de TB por cada 100 mil habitantes en población general y, el $72 \%$ de estos casos se reportaron en seis departamentos del país ${ }^{(15)}$; lo que pone a los TS en constante riesgo de adquirir la infección y enfermedad tuberculosa en los centros laborales o en la comunidad. En los últimos 20 años varios estudios en el país han reportado altas tasas de prevalencia de infección tuberculosa latente en TS, tanto de hospitales y ES del primer nivel de atención así como en estudiantes e internos de carreras de ciencias de la salud ${ }^{(16-21)}$.

Los estudios sobre TB en TS reportados en Perú, se han basado principalmente en reporte de casos de hospitales nacionales, algunos de varios años de seguimiento y otros como reportes de brotes ${ }^{(17,22-25)}$. Las estadísticas nacionales reportaron más de mil casos de TB en TS con un promedio de 200 casos por año y una tendencia creciente entre los años 2007 al $2012^{(26) ;}$ sin embargo, solo se realizaban reportes consolidados que no permitían el análisis de las características de la enfermedad en este grupo ocupacional. El año 2013 se implementó en Perú la vigilancia epidemiológica de la tuberculosis (VTB), incluyendo la notificación obligatoria de los casos de TB en trabajadores de salud, definida como la notificación individual de todo caso de TB en profesional, técnico $u$ otro personal que labore en un establecimiento de salud (ES). Al año 2015, la VTB se encuentra implementada en todos los ES públicos y privados del país que conforman la Red Nacional de Epidemiologia que, para este año. fueron 8565 ES (incluyendo los establecimientos del Ministerio de Salud (MINSA), los asignados al Instituto de Gestión de Servicios de Salud, de los gobiernos regionales, de EsSalud, de las Fuerzas Armadas (FF.AA.), de la Policía Nacional del Perú (PNP), los del Instituto Nacional Penitenciario (INPE) y los privados.
El presente estudio tiene por objetivo describir las características epidemiológicas de la TB en trabajadores de salud y estimar la incidencia de enfermedad en este grupo ocupacional, utilizando la información de la VTB.

\section{MATERIALES Y MÉTODOS}

Se realizó un estudio descriptivo que incluyó a todos los trabajadores de salud afectados por tuberculosis notificados al sistema nacional de vigilancia epidemiológica del MINSA del Perú, durante los años 2013, 2014 y 2015. Se revisó la base de datos de la VTB, obteniéndose las siguientes variables por su relevancia epidemiológica: edad, sexo, órgano afectado, baciloscopía de diagnóstico, profesión, condición laboral, servicio, establecimiento donde labora y establecimiento notificante.

La VTB se basa en la notificación sistemática de casos con diagnóstico de tuberculosis, para cada caso se completa una ficha epidemiológica, la misma que es llenada por el personal de salud del establecimiento que realiza el diagnóstico; la ficha epidemiológica es registrada en el aplicativo electrónico SIEPI-TB que es una herramienta informática, que funciona en tiempo real, desarrollada en plataforma web y alojada en el servidor del Centro Nacional de Epidemiologia, Prevención y Control de Enfermedades (ex Dirección General de Epidemiologia). El ingreso de la información al aplicativo electrónico se realiza en el ES que es unidad notificante, y los lugares donde no se tiene acceso a Internet lo realiza la red, microrred o la dirección regional de salud a la que pertenece el ES notificante. Cada ES notificante y la dirección regional de salud tienen acceso a la información de sus casos, a actualizar los datos, a la generación de reportes y a la descarga de su base de datos con fines de realizar control de calidad y análisis. El personal responsable de la VTB del MINSA, realiza asistencia técnica y el monitoreo de la calidad de la información de manera semanal.

Se realizó un análisis descriptivo basado en la obtención de frecuencias, porcentajes, medidas de tendencia central y de dispersión. La incidencia de la tuberculosis en TS se estimó con el número de casos reportados al sistema de vigilancia y la información de recursos humanos por año, por ocupación y por establecimiento de salud brindado por el Observatorio de Recursos Humanos en Salud del Perú, de la Dirección General de Gestión del Desarrollo de Recursos Humanos del MINSA. El análisis estadístico fue realizado con el programa estadístico STATA versión 11.0.

Con relación a los aspectos éticos, se utilizó una fuente secundaria, los datos notificados al Sistema Nacional de Vigilancia Epidemiológica del MINSA, la base de datos 
de la vigilancia de tuberculosis se obtuvo omitiéndose los nombres y DNI de los pacientes, a fin de garantizar la confidencialidad de la información, y se contó con la autorización institucional para la difusión de los datos.

\section{RESULTADOS}

Se notificaron mediante la VTB un total de 755 casos de TB en TS de todo el país (226 casos en el año 2013; 241 en el año 2014 y 288 en el año 2015). Del total de casos notificados, el 65\% (493 casos) laboraban en el MINSA, 20\% (153 casos) en EsSalud, 3\% (21 casos) en las FF.AA., PNP e INPE y $12 \%$ (88 casos) en establecimientos privados. No todos los casos se notificaron por el establecimiento donde laboraba el TS, en el periodo de estudio encontramos que el $60,4 \%$ (456/755) de los casos fueron reportados por el mismo establecimiento donde laboraba el TS, este hecho fue más frecuente en establecimientos de EsSalud donde se reportó $80,4 \%(123 / 153)$ y menos frecuente en establecimientos privados, donde solo se reportó $1,1 \%(1 / 88)$, en establecimientos de salud del MINSA se reportó un $64,9 \%$ (320/493) y en establecimientos de salud de las FF.AA., PNP e INPE un 61,9\% (12/21). Del total de casos notificados por ES del MINSA (493), el $77,3 \%$ fueron notificados por ES del MINSA y el $22,7 \%$ por ES de EsSalud, Tabla 1.

El $63,6 \%$ de los casos de TB en TS fueron mujeres y la edad promedio fue 38 años con un rango de 19 a 89 años, las mujeres reportaron un promedio de edad menor (37 años) que el de los hombres (casi 41 años).

El $57,0 \%$ de los casos de TB en TS se concentró en Lima Metropolitana y el Callao, ciudades que reportan casi el $60 \%$ de los casos de TB en población en general. El mayor porcentaje de TS afectados fueron casos nuevos, solo el $8,5 \%$ de los casos reportaron tener antecedente de recibir tratamiento antituberculosis; en la población general fue mayor el porcentaje de casos antes tratados $(13,2 \%)$ y esta diferencia fue significativa. $\mathrm{El}$ antecedente de contacto fue mayor en TS que en población general $(47,4$ vs $32,8 \%, p<0,001)$. Se reportó un $6 \%$ de casos resistentes, principalmente casos de TB MDR. Los casos de TB pulmonar con confirmación bacteriológica en TS fue $67,0 \%$ y la mitad de estos casos fueron diagnosticados con carga bacilar alta $(2+$ y $3+$ ). El porcentaje de TB pulmonar sin confirmación bacteriológica y TB extrapulmonar fue 10\% mayor en TS que en población general y esta diferencia fue significativa (Tabla 2).

Entre profesionales y técnicos de la salud sumaron el $82,6 \%$ de los casos reportados y $14,6 \%$ fueron personal administrativo, los técnicos en enfermería, médicos y enfermeros fueron los grupos ocupacionales más afectados. El vínculo laboral más frecuente fue el ser contratado $(47,4 \%)$. Entre estudiantes e internos de carreras de salud se reportaron $7,9 \%$ del total de casos; sin embargo, los internos afectados fueron estudiantes de carreras profesionales de la salud, llegando a representar 9,2\% (36/392) de los profesionales de la Salud. En médicos residentes se reportó 3,7\% casos, pero estos representaron un $20 \%(28 / 137)$ del total de médicos afectados. El $61,6 \%$ de los casos laboraban en hospitales, un menor porcentaje $(25,8 \%)$ en establecimientos del primer nivel de atención y $12,6 \%$ en establecimientos privados, las áreas clínicas de consultorios, hospitalización y emergencia fueron las áreas donde laboraban más del $50 \%$ de los casos en general (Tabla 3).

Se observó diferencias significativas por niveles de atención, en hospitales fue alto el porcentaje de afectados procedentes de Lima y Callao $(64 \%)$ y en ES del primer nivel de atención mayor el porcentaje procedente del resto de provincias del país $(63,1 \%)$ $y$ esto fue estadísticamente significativo $(p<0,001)$, para hospitales, las áreas donde laboraban el mayor porcentaje de TS afectados fueron consultorios, hospitalización y áreas administrativas, para TS de ES del primer nivel de atención fueron consultorios, programa de tuberculosis y emergencia; además, se observaron diferencias significativas para las áreas de consultorio $(p<0,001)$, Hospitalización $(p<0,001)$,

Tabla 1. Casos de tuberculosis en trabajadores de salud por instituciones que notifican y laboran, Perú años 2013-2015

\begin{tabular}{|c|c|c|c|c|c|c|c|c|c|c|c|c|}
\hline & \multicolumn{2}{|c|}{$\begin{array}{c}\text { Institución } \\
\text { donde laboran }\end{array}$} & \multicolumn{2}{|c|}{$\begin{array}{l}\text { Casos } \\
\text { notificados por } \\
\text { el mismo ES }\end{array}$} & \multicolumn{2}{|c|}{$\begin{array}{c}\text { Casos que se } \\
\text { notificaron en ES } \\
\text { de MINSA }\end{array}$} & \multicolumn{2}{|c|}{$\begin{array}{l}\text { Casos que se } \\
\text { notificaron en ES } \\
\text { de EsSalud }\end{array}$} & \multicolumn{2}{|c|}{$\begin{array}{l}\text { Casos que se } \\
\text { notificaron en ES } \\
\text { FF.AA./PNP/INPE }\end{array}$} & \multicolumn{2}{|c|}{$\begin{array}{c}\text { Casos que se } \\
\text { notificaron en } \\
\text { ES privados }\end{array}$} \\
\hline & $\mathrm{n}$ & (\%) & $\mathrm{n}$ & (\%) & $\mathrm{n}$ & (\%) & $\mathrm{n}$ & (\%) & $\mathrm{n}$ & (\%) & $\mathrm{n}$ & (\%) \\
\hline \multicolumn{13}{|l|}{ Institución } \\
\hline MINSA & 493 & $(65,0)$ & $320 / 493$ & $(64,9)$ & $381 / 493$ & $(77,3)$ & $112 / 493$ & $(22,7)$ & 0,0 & & 0,0 & \\
\hline EsSalud & 153 & (20) & 123/153 & $(80,4)$ & $11 / 153$ & $(7,2)$ & $142 / 153$ & $(92,8)$ & 0,0 & & 0,0 & \\
\hline $\begin{array}{l}\text { FF.AA./PNP/ } \\
\text { INPE }\end{array}$ & 21 & (3) & $12 / 21$ & $(61,9)$ & $5 / 21$ & $(23,8)$ & $3 / 21$ & $(14,3)$ & $13 / 21$ & $(61,9)$ & 0,0 & \\
\hline Privado & 88 & (12) & $1 / 88$ & $(1,1)$ & $50 / 88$ & $(56,8)$ & $37 / 88$ & (42) & 0,0 & & $1 / 88$ & $(1,1)$ \\
\hline Total & 755 & & $56 / 755$ & $(60,4)$ & $447 / 755$ & $(59,2)$ & $294 / 755$ & $(38,9)$ & $13 / 755$ & $(1,7)$ & $1 / 755$ & $(0,1)$ \\
\hline
\end{tabular}


Tabla 2. Características epidemiológicas y clínicas de trabajadores de salud diagnosticados con tuberculosis comparado con población general. Perú 2013-2015

\begin{tabular}{|c|c|c|c|c|c|}
\hline & \multicolumn{2}{|c|}{$\begin{array}{c}\text { Trabajadores de salud } \\
n=755\end{array}$} & \multicolumn{2}{|c|}{$\begin{array}{c}\text { Población general } \\
n=75946\end{array}$} & \multirow[b]{2}{*}{ Valor $p$} \\
\hline & frecuencia & $(\%)$ & frecuencia & $(\%)$ & \\
\hline Edad* $^{*}$ & &, $1( \pm 12,3)$ & & $35,9( \pm 18,8)$ & $<0,001$ \\
\hline \multicolumn{6}{|l|}{ Sexo } \\
\hline Masculino & 275 & $(36,4)$ & & $(64,1)$ & $<0,001$ \\
\hline Femenino & 480 & $(63,6)$ & & $(35,9)$ & \\
\hline \multicolumn{6}{|l|}{ Lugar de procedencia } \\
\hline Lima Metropolitana y Callao & 430 & $(57,0)$ & & $(60,7)$ & 0,036 \\
\hline Otros & 325 & $(43,0)$ & & $(39,3)$ & \\
\hline \multicolumn{6}{|l|}{ Antecedente de contacto } \\
\hline Sí & 358 & $(47,4)$ & & $(32,8)$ & $<0,001$ \\
\hline \multicolumn{6}{|l|}{ Antecedente de tratamiento } \\
\hline Nuevos & 691 & $(91,5)$ & & $(86,8)$ & $<0,001$ \\
\hline Antes tratados & 64 & $(8,5)$ & & $(13,2)$ & \\
\hline Casos resistentes & 46 & $(6,1)$ & & $(9,3)$ & 0,002 \\
\hline TB MDR & 31 & $(4,1)$ & & $(5,1)$ & 0,216 \\
\hline TB XDR & 2 & $(0,3)$ & & $(0,4)$ & 0,557 \\
\hline TB poliresistente o monoresistente & 13 & $(1,7)$ & & $(3,8)$ & 0,003 \\
\hline \multicolumn{6}{|l|}{ Localización de la tuberculosis } \\
\hline Pulmonar & 545 & $(72,2)$ & & $(82,2)$ & $<0,001$ \\
\hline Extrapulmonar & 210 & $(27,8)$ & & $(17,8)$ & \\
\hline \multicolumn{6}{|c|}{$\begin{array}{l}\text { Confirmación bacteriológica y carga bacilar en los } \\
\text { casos de TB pulmonar }\end{array}$} \\
\hline Sin confirmación bacteriológica & $180 / 545$ & $(33,0)$ & 62426 & $(23,2)$ & $<0,001$ \\
\hline Confirmación bacteriológica & $365 / 545$ & $(67,0)$ & 62426 & $(76,8)$ & \\
\hline Positivo (+) & $186 / 365$ & $(51,0)$ & 47943 & $(45,2)$ & 0,003 \\
\hline Positivo $(++)$ & $104 / 365$ & $(28,5)$ & 47943 & $(26,3)$ & \\
\hline Positivo (+++) & $75 / 365$ & $(20,5)$ & 47943 & $(28,5)$ & \\
\hline \multicolumn{6}{|c|}{ Confirmación bacteriológica en las TB extrapulmonar } \\
\hline Con confirmación bacteriológica & $10 / 210$ & $(4,8)$ & 13520 & $(8,8)$ & 0,040 \\
\hline Sin confirmación bacteriológica & $200 / 210$ & $(95,2)$ & 13520 & $(91,2)$ & \\
\hline \multicolumn{6}{|l|}{ Localización extrapulmonar } \\
\hline Pleural & $90 / 210$ & $(42,9)$ & 13520 & $(53,2)$ & $<0,001$ \\
\hline Mamas & $25 / 210$ & $(11,9)$ & 13520 & $(5,5)$ & \\
\hline Ganglionar & $24 / 210$ & $(11,4)$ & 13520 & $(11,1)$ & \\
\hline Piel & $12 / 210$ & $(5,7)$ & 13520 & $(2,3)$ & \\
\hline Sistema nervioso & $12 / 210$ & $(5,7)$ & 13520 & $(9,0)$ & \\
\hline Otros & $47 / 210$ & $(22,4)$ & 13520 & $(18,9)$ & \\
\hline
\end{tabular}

Programa de TB $(p<0,001)$ y áreas administrativas (con $p=0,001$ ). Respecto a las ocupaciones para hospitales, los técnicos de enfermería, médicos y personal administrativo fueron los grupos ocupacionales más afectados, para ES del primer nivel de atención, los enfermeros y técnicos de enfermería fueron los grupos ocupacionales más afectados, se observaron diferencias significativas para el grupo de enfermeras $(p=0,004)$, obstetras $(p<0,001)$, odontólogos $(p=0,003)$ y ocupaciones administrativas $(p<0,001)$ (Tabla 4$)$. 
Tabla 3. Características de las condiciones laborales de trabajadores de salud diagnosticados con tuberculosis, Perú 2013-2015 ( $\mathrm{n}=755)$

\begin{tabular}{|c|c|c|}
\hline & $n=755$ & $(\%)$ \\
\hline \multicolumn{3}{|l|}{ Ocupación } \\
\hline Profesionales de la salud ${ }^{*}$ & 392 & $(51,9)$ \\
\hline Médico & 137 & $(18,1)$ \\
\hline Enfermero & 144 & $(19,1)$ \\
\hline Obstetra & 31 & $(4,1)$ \\
\hline Odontólogo & 22 & $(2,9)$ \\
\hline Otros profesionales ${ }^{* *}$ & 58 & $(7,7)$ \\
\hline Técnicos de salud & 232 & $(30,7)$ \\
\hline Técnico en enfermería & 190 & $(25,2)$ \\
\hline Otros técnicos ${ }^{* \star *}$ & 42 & $(5,6)$ \\
\hline Administrativos & 110 & $(14,6)$ \\
\hline Profesional administrativo & 5 & $(0,7)$ \\
\hline Asistentes administrativos & 48 & $(6,4)$ \\
\hline Trabajadores de limpieza & 29 & $(3,8)$ \\
\hline Personal de vigilancia & 8 & $(1,1)$ \\
\hline Ocupaciones elementales (chofer, personal de mantenimiento y lavandería) & 20 & $(2,6)$ \\
\hline No se precisa & 22 & $(2,9)$ \\
\hline \multicolumn{3}{|l|}{ Vínculo laboral } \\
\hline Contratado (CAS + terceros) & 358 & $(47,4)$ \\
\hline Nombrado & 214 & $(28,3)$ \\
\hline \multicolumn{3}{|l|}{ Grupos especiales } \\
\hline Interno & 36 & $(4,8)$ \\
\hline Residente médico & 28 & $(3,7)$ \\
\hline Estudiante & 24 & $(3,2)$ \\
\hline SERUMS & 11 & $(1,5)$ \\
\hline Privado, particular o independiente & 33 & $(4,4)$ \\
\hline No se precisa & 51 & $(6,8)$ \\
\hline \multicolumn{3}{|l|}{ Niveles de establecimientos donde laboran } \\
\hline Hospitales & 465 & $(61,6)$ \\
\hline Establecimientos del primer nivel de atención & 195 & $(25,8)$ \\
\hline Establecimientos privados & 95 & $(12,6)$ \\
\hline \multicolumn{3}{|l|}{ Áreas donde labora en el establecimiento de salud } \\
\hline Áreas clínicas & 485 & $(64,2)$ \\
\hline Consultorios & 215 & $(28,5)$ \\
\hline Hospitalización & 117 & $(15,5)$ \\
\hline Centro quirúrgico & 17 & $(2,3)$ \\
\hline Emergencia & 85 & $(11,3)$ \\
\hline Programa de tuberculosis & 51 & $(6,8)$ \\
\hline Laboratorio & 27 & $(3,6)$ \\
\hline Farmacia & 31 & $(4,1)$ \\
\hline Áreas administrativas & 138 & $(18,3)$ \\
\hline Áreas de nutrición/cocina & 6 & $(0,8)$ \\
\hline Áreas administrativas de atención al público & 22 & $(2,9)$ \\
\hline Oficinas administrativas & 58 & $(7,7)$ \\
\hline Áreas de servicio y mantenimiento & 52 & $(6,9)$ \\
\hline Varias áreas & 22 & $(2,9)$ \\
\hline No se precisa & 52 & $(6,9)$ \\
\hline Total & 755 & $100 \%$ \\
\hline
\end{tabular}

* Incluyen residentes de medicina, internos y estudiantes

** Incluye asistenta social, biólogo, Lic. Rehabilitación, Lic. Nutrición, psicólogo, químico farmacéutico y tecnólogo médico

*** Incluye técnico de farmacia, de laboratorio, de nutrición, dental y técnico radiólogo 
Tabla 4. Características de las condiciones laborales de trabajadores de salud diagnosticados con tuberculosis según nivel de atención, Perú 2013-2015 (n=660)

\begin{tabular}{|c|c|c|c|c|c|}
\hline & \multicolumn{2}{|c|}{ Hospitales } & \multicolumn{2}{|c|}{$\begin{array}{l}\text { Establecimientos } \\
\text { del primer nivel }\end{array}$} & \multirow[b]{2}{*}{ Valor $p^{\star *}$} \\
\hline & $\mathrm{N}=465$ & $(\%)$ & $\mathrm{N}=195$ & (\%) & \\
\hline \multicolumn{6}{|l|}{$\begin{array}{l}\text { Lugar donde } \\
\text { laboran }\end{array}$} \\
\hline $\begin{array}{l}\text { Lima } \\
\text { Metropolitana y } \\
\text { Callao }\end{array}$ & 301 & $(64,7)$ & 72 & $(36,9)$ & $<0,001$ \\
\hline Otros & 164 & $(35,3)$ & 123 & $(63,1)$ & \\
\hline \multicolumn{6}{|l|}{$\begin{array}{l}\text { Áreas donde } \\
\text { laboran }\end{array}$} \\
\hline Consultorios & 99 & $(21,3)$ & 75 & $(38,5)$ & $<0,001$ \\
\hline $\begin{array}{l}\text { Áreas de } \\
\text { hospitalización/ } \\
\text { centro } \\
\text { quirúrgico }\end{array}$ & 127 & $(27,3)$ & 0 & $(0,0)$ & $<0,001$ \\
\hline Emergencia & 59 & $(12,7)$ & 21 & $(10,8)$ & 0,491 \\
\hline $\begin{array}{l}\text { Programa de } \\
\text { tuberculosis }\end{array}$ & 14 & $(3,0)$ & 37 & $(19,0)$ & $<0,001$ \\
\hline Laboratorio & 17 & $(3,7)$ & 3 & $(1,5)$ & 0,148 \\
\hline Farmacia & 12 & $(2,6)$ & 7 & $(3,6)$ & 0,479 \\
\hline $\begin{array}{l}\text { Áreas } \\
\text { administrativas }\end{array}$ & 100 & $(21,5)$ & 20 & $(10,3)$ & 0,001 \\
\hline Varias áreas & 12 & $(2,6)$ & 12 & $(6,2)$ & 0,025 \\
\hline No se precisa & 25 & $(5,4)$ & 20 & $(10,3)$ & 0,023 \\
\hline \multicolumn{6}{|l|}{ Ocupación } \\
\hline $\begin{array}{l}\text { Profesionales de la } \\
\text { salud }^{*}\end{array}$ & 226 & $(48,6)$ & 124 & $(63,6)$ & \\
\hline Médico & 97 & $(20,9)$ & 28 & $(14,4)$ & 0,052 \\
\hline Enfermero & 81 & $(17,4)$ & 53 & $(27,2)$ & 0,004 \\
\hline Obstetra & 10 & $(2,2)$ & 17 & $(8,7)$ & $<0,001$ \\
\hline Odontólogo & 7 & $(1,5)$ & 11 & $(5,6)$ & 0,003 \\
\hline $\begin{array}{l}\text { Otros } \\
\text { profesionales }\end{array}$ & 31 & $(6,7)$ & 15 & $(7,7)$ & \\
\hline Técnicos de salud & 139 & $(29,9)$ & 52 & $(26,7)$ & \\
\hline $\begin{array}{l}\text { Técnico en } \\
\text { enfermería }\end{array}$ & 120 & $(25,8)$ & 45 & $(23,1)$ & 0,460 \\
\hline $\begin{array}{l}\text { Otros } \\
\text { técnicos*** }\end{array}$ & 19 & $(4,1)$ & 7 & $(3,6)$ & \\
\hline Administrativos & 89 & $(19,1)$ & 15 & $(7,7)$ & $<0,001$ \\
\hline No se precisa & 11 & $(2,4)$ & 4 & $(2,1)$ & \\
\hline
\end{tabular}

* Incluyen residentes de Medicina, internos y estudiantes

** Incluye asistenta social, biólogo, Lic. Rehabilitación, Lic. Nutrición, psicólogo, químico farmacéutico y tecnólogo médico

*** Incluye técnico de farmacia, de laboratorio, de nutrición, dental y técnico radiólogo

Incluye profesional administrativo, asistente administrativo, trabajadores de limpieza, personal de vigilancia, chofer, personal de mantenimiento y lavandería

Para los años 2013, 2014 y 2015 se estimó una incidencia de TB en TS en general de 96,1; 91,8 y 110,3 casos nuevos de TB por cada 100 mil TS, respectivamente. La incidencia de TB en los grupos ocupacionales más afectados (médicos, enfermeros y técnicos en enfermería) fue mayor de lo observado en TS total (Tabla 5). La incidencia específica en los
Tabla 5. Incidencia de tuberculosis en trabajadores de salud a nivel nacional y por grupos ocupacionales. Perú 2013-2015

\begin{tabular}{lccc}
\hline & \multicolumn{3}{c}{ Incidencia *100000 } \\
\cline { 2 - 4 } & $\mathbf{2 0 1 3}$ & $\mathbf{2 0 1 4}$ & $\mathbf{2 0 1 5}$ \\
\hline Total de trabajadores de salud & 96,1 & 91,8 & 110,3 \\
$\begin{array}{l}\text { Profesional y/o técnicos de } \\
\text { salud asistencial }\end{array}$ & 98,7 & 106,1 & 129,1 \\
$\begin{array}{l}\text { Profesional y/o técnico } \\
\text { administrativo }\end{array}$ & 60,0 & 59,2 & 53,7 \\
Médico & 124,7 & 117,5 & 159,2 \\
Enfermera & 115,1 & 104,6 & 145,1 \\
\hline Técnico enfermería & 127,7 & 140,1 & 186,9 \\
\hline
\end{tabular}

* por 100000 habitantes

diez hospitales que reportaron el mayor número de TS afectados fue de 2 a 8 veces mayor que el observado en TS a nivel de país (con tasas entre 167 a 833 por 100 mil TS para el año 2013, 193 a 440 para el año 2014 y 178 a 571 para el año 2015).

\section{DISCUSIÓN}

El presente estudio muestra un incremento de casos de TB en trabajadores de salud, donde un alto porcentaje de afectados son técnicos de enfermería, enfermeras y médicos que laboraban en hospitales. Según los niveles de atención se evidencia un patrón característico y diferente para TS procedentes de hospitales y para TS procedentes de ES del primer nivel de atención, resaltando que los primeros son procedentes en mayor proporción de ES de Lima y Callao, y los segundos de ES de provincias, además, las áreas de consultorios y el programa de tuberculosis fueron las áreas que concentraron a la mitad de los casos, diferente a lo reportado en hospitales donde esa proporción de casos laboraban en áreas de hospitalización y consultorios.

El riesgo de exposición y el desarrollo de la infección tuberculosa latente son dos fases importantes en el desarrollo de la enfermedad tuberculosa en los TS, la probabilidad de ponerse en contacto con un paciente bacilífero no diagnosticado que acude a un hospital ubicado en una zona endémica de TB es alta ${ }^{(2)}$. Un estudio realizado en el Perú encontró $21 \%$ de enfermedad tuberculosa $(71 \%$ de los cuales fueron bacilíferos) en pacientes que acudieron y permanecieron más de dos horas en el servicio de emergencia de un hospital (16); por otro lado, varios estudios reportaron prevalencias de infección tuberculosa latente en TS superiores a $50 \%{ }^{(16-19)}$.

La incidencia anual de TB en TS reportada en el presente estudio, fue estimada con el total de TS del país por año, el valor estimado fue casi el doble de lo reportado en población general ${ }^{(15)}$, además, se observa una variación 
y un incremento relevante por grupos ocupacionales, igualmente, la incidencia en los ES que reportan el mayor número de casos fue hasta ocho veces más que lo reportado en población, algunos estudios estimaron incidencias similares para hospitales nacionales. Echanove et al. ${ }^{(17)}$ reportaron una tasa superior a 700 casos por 100 mil TS en el año 1997, con un incremento de hasta siete veces al reportado inicialmente en un periodo de 4 años, por otro lado, Danilla reportó una tasa de 520 por 100 mil TS en el año 2003 para otro hospital nacional ${ }^{(22)}$

Las áreas o ambientes donde laboran los TS constituyen un riesgo en la trasmisión y desarrollo de la enfermedad, en países de medianos y bajos ingresos se describió las áreas de hospitalización TB, laboratorios, salas de medicina general y las salas de emergencia como áreas de riesgo alto para contraer $\mathrm{TB}$, los servicios ambulatorios (consultorios) se clasificaron con riesgo intermedio y, las áreas de cirugía, obstetricia, quirófano y administrativas como bajo riesgo ${ }^{(4)}$. El presente estudio reportó que un tercio de TS afectados por TB laboraban en áreas de consultorio y servicios ambulatorios, esta proporción fue mayor para ES del primer nivel de atención y establecimientos de salud privados, probablemente porque en estos establecimientos las consultas externas son el servicio que más se brinda. En hospitales, las áreas de hospitalización y emergencia concentraron al $40 \%$ de los casos; en estas áreas, el contacto con pacientes es más prolongado y no siempre se toman las medidas de protección, en especial en áreas de hospitalización.

A pesar de que en otro estudio ${ }^{(4)}$ se reportan las áreas de laboratorio como de alto riesgo, en el presente estudio se reportó menos de $4 \%$ de casos en esta área. Así también, se reportaron más casos en áreas consideradas de bajo riesgo, tales como áreas administrativas, farmacia, áreas de nutrición o cocina y áreas de mantenimiento del ES. Si bien en zonas endémicas estos casos podrían ser de trasmisión en comunidad más que en los ambientes hospitalarios, es importante identificar los riesgos hospitalarios que podrían contribuir con la trasmisión de la TB en estas áreas, por ejemplo aquellas que brindan atención al público, como admisión, farmacias o de nutrición que, en muchos casos, reparten los alimentos por todos los ambientes del hospital, probablemente estos factores podrían incrementar el riesgo de exposición.

En las áreas donde se brinda el tratamiento y seguimiento a pacientes ya diagnosticados (de elevado riesgo de exposición) ${ }^{(4)}$, nuestro estudio reportó un $6,8 \%$ de TS con TB, sin embargo, el porcentaje fue tres veces mayor en ES del primer nivel de atención que en hospitales, la explicación a este hallazgo se puede relacionar con las políticas establecidas en el país, donde la mayoría de hospitales diagnostican a los casos (por lo general en áreas de consultorio, hospitalización y emergencia), pero son los establecimientos de salud del primer nivel de atención los que brindan el tratamiento y realizan el seguimiento de los casos ya diagnosticados.

Respecto a la ocupación, la identificación de grupos de riesgo ha sido muy heterogénea en los diferentes estudios $^{(2-4)}$. En el estudio se encontró que casi $2 / 3$ de los casos de TB fueron técnicos de enfermería, enfermeras y médicos. Si bien este hallazgo podría estar vinculado al rol que realizan en la atención de los pacientes, a los ambientes donde laboran y a la aplicación de medidas de control de infecciones y bioseguridad, un factor que no fue evaluado en el presente estudio, pero que influye en el desarrollo de la enfermedad tuberculosa, son los riesgos individuales que cada persona tendría para contraer la enfermedad, entre ellos las condiciones o comorbilidades que genera disminución de la inmunidad. Es importante conocer y evaluar el riesgo individual de los trabajadores que presentan infección tuberculosa latente, que trabajan en áreas de riesgo elevado, tienen mayor edad y mayor tiempo de servicio, estando esta población en mayor riesgo de desarrollar enfermedad ${ }^{(2-4)}$.

Al igual que otros reportes de trabajadores de salud con TB ${ }^{(23-25)}$, observamos un gran porcentaje de afectados con vínculo laboral contratado, una situación particular dado que según las estadísticas del Observatorio de Recursos Humanos en Salud de Perú, un poco más de la mitad de los TS tiene vínculo laboral nombrado, y solo un tercio del personal es contratado, probablemente este resultado esté vinculado también a los grupos ocupacionales más afectados. Por otro lado, aunque el porcentaje de casos en internos y estudiantes de carreras de salud fue menor al $10 \%$, estos, en su mayoría, fueron de carreras profesionales de medicina y enfermería, este porcentaje podría ser mayor al reportado, dado que este grupo no tienen un vínculo laboral estable con la institución y, muchas veces, no son considerados como trabajadores de salud de la institución, pero desarrollan actividades de formación de corto tiempo (un año o menos) en los establecimientos de salud y están en constante exposición a la enfermedad. Accineli et al. ${ }^{(23)}$, reportaron un $36,6 \%$ de estudiantes de ciencias de la salud afectados por TB en un hospital nacional, en un periodo de seguimiento de casi 15 años, donde el $87 \%$ de estos fueron estudiantes de Medicina. Los médicos residentes representaron una quinta parte de los médicos afectados por TB; este grupo presenta características particulares por estar en formación para una especialidad, permanecen muchas horas a lo largo de su formación en las diferentes instalaciones de un hospital y muchas veces por sus actividades académicas y laborales no duermen muchas horas o no se alimentan adecuadamente, lo que les genera mayores condiciones para desarrollar la enfermedad.

El presente estudio reportó un mayor porcentaje de TS mujeres con un promedio de edad mayor que lo 
reportado para población general (donde mayor es el porcentaje de afectados varones con promedio de edad menor), al respecto se ha descrito ${ }^{(2-4)}$ mayor edad y mayor tiempo de servicio como factores de riesgo importantes en el desarrollo de la infección y enfermedad tuberculosa en TS, por otro lado, el mayor porcentaje de mujeres afectadas estaría relacionado con los grupos ocupacionales más afectados, ya que el porcentaje de mujeres es alto en las carreras de enfermería y técnicos en enfermería.

Las características clínicas y de diagnóstico de TB en TS reportadas en el presente estudio, muestran un patrón diferente al de población general, resaltando que se presentan un mayor porcentaje de casos de TB pulmonar con baciloscopía negativa y mayor porcentaje de TB extrapulmonar que la población general, probablemente relacionada con la percepción de enfermedad en los TS o la búsqueda activa de contactos cuando se detecta un caso en el ES; sin embargo, sería importante realizar más investigaciones sobre este patrón característico, que también se ha reportado en otros estudios ${ }^{(22-25)}$.

Una limitación del estudio se relaciona con el subregistro en la notificación de casos al sistema de vigilancia epidemiológica, relacionada probablemente al estigma social que todavía genera la tuberculosis, aun más en trabajadores de salud, motivo por el cual no todos los casos de TB en TS, se identifican como tales para ser notificados al sistema de vigilancia, o simplemente no llegan a ser captados por el programa de control o se automedican; sin embargo, aun así, la vigilancia epidemiológica de tuberculosis registró más casos que lo reportado por el informe operacional del programa de control, en el que se recolecta de manera consolidada el número de TS por el ES donde labora el TS con una periodicidad trimestral ${ }^{(15)}$. Un hallazgo importante del presente estudio fue el alto porcentaje de casos de TS con TB en establecimientos de salud privados y casi el $100 \%$ de ellos captados y notificados por un establecimiento de MINSA o EsSalud. Otra limitación del estudio relacionada con el uso de datos secundarios es el error de registro y la limitación de información específica para ampliar el análisis de algunas variables como por ejemplo, no se pudo identificar los cargos que desempeñan los trabajadores, que muchas veces no se relaciona con la ocupación o el área donde laboran, por otro lado tampoco se pudo identificar las áreas específicas de hospitalización y consultorios donde laboraban los afectados, ni los tiempos que laboraran en los áreas de trabajo, o las constantes rotaciones de personal por áreas que se dan en muchos establecimientos de salud. A pesar de estas limitaciones, resaltamos que este es el primer estudio que reporta la problemática de la TB en TS a nivel nacional tomando como fuente la vigilancia epidemiológica de tuberculosis, mostrando información relevante y útil para las acciones de prevención y control en los establecimientos de salud de todos los niveles de atención.

La TB constituye un riesgo laboral importante para los TS de los establecimientos de salud públicos y privados de nuestro país, afecta principalmente al grupo de profesionales y técnicos de la salud, que realizan labores en áreas de cuidado y atención directa con pacientes en grandes hospitales del país, las características de las condiciones laborales son diferentes para trabajadores de hospitales y los trabajadores de salud del primer nivel de atención.

Agradecimientos: a todo el personal de salud de las unidades notificantes e informantes de la Red Nacional de Epidemiologia, a los directores de Epidemiologia y responsables de la vigilancia de tuberculosis de las direcciones regionales de salud, por el trabajo realizado en el proceso de implementación de la vigilancia de tuberculosis, su esfuerzo permite generar información para un mejor conocimiento del estado actual y evolución de la tuberculosis en nuestro país.

Contribuciones de los autores: MS y MY participaron en la concepción y diseño del estudio, MS y AC participaron en la recolección de los datos, todos los autores participaron en el análisis e interpretación de los resultados. JA y MY participaron en la revisión crítica del artículo MS y MY participaron en la redacción del artículo. Todos los autores aprobaron la versión final a publicar.

Fuentes de financiamiento: Dirección General de Epidemiología, actual Centro Nacional de Epidemiologia, Prevención y Control de Enfermedades del Ministerio de Salud, Lima, Perú.

Conflictos de interés: MS, AC y JA son trabajadores del Centro Nacional de Epidemiología, Prevención y Control de Enfermedades del Ministerio de Salud.

\section{REFERENCIAS BIBLIOGRÁFICAS}

1. List of occupational diseases (revised 2010). Identification and recognition of occupational diseases: Criteria for incorporating diseases in the ILO list of occupational diseases [Internet]. 2010 [citado 10 de marzo de 2016]. Recuperado a partir de: http://www. ilo.org/safework/info/publications/ WCMS_150323/lang--en/index.htm
2. Baussano I, Nunn P, Williams B, Pivetta E, Bugiani M, Scano F. Tuberculosis among health care workers. Emerg Infect Dis. marzo de 2011;17(3):488-94.

3. Menzies D, Joshi R, Pai M. Risk of tuberculosis infection and disease associated with work in health care settings. Int J Tuberc Lung Dis Off J
Int Union Tuberc Lung Dis. junio de 2007;11(6):593-605.

4. Joshi R, Reingold AL, Menzies D, Pai M. Tuberculosis among health-care workers in low- and middle-income countries: a systematic review. PLoS Med. diciembre de 2006;3(12):e494.

5. Casas I, Esteve M, Guerola R, García-Olivé I, Roldán-Merino J, 
Martinez-Rivera C, et al. Incidence of tuberculosis infection among healthcare workers: risk factors and 20-year evolution. Respir Med. abril de 2013;107(4):601-7.

6. Perfecto B, Sánchez JR, González AI, López I, Dorronsoro I. Outbreak of multiresistant tuberculosis. An Sist Sanit Navar. agosto de 2000;23(2):257-63.

7. Huang H-Y, Jou R, Chiang C-Y, Liu W-C, Chiu H-J, Lee J-J. Nosocomial transmission of tuberculosis in two hospitals for mentally handicapped patients. J Formos Med Assoc Taiwan Yi Zhi. diciembre de 2007;106(12):999-1006.

8. Saleiro S, Rosa Santos A, Vidal O, Carvalho T, Torres Costa J, Agostinho Marques J. Tuberculosis in hospital department health care workers. Rev Port Pneumol Engl Ed. noviembre de 2007;13(6):789-99.

9. Tudor C, Van der Walt M, Margot B, Dorman SE, Pan WK, Yenokyan G, et al. Tuberculosis among health care workers in KwaZulu-Natal, South Africa: a retrospective cohort analysis. BMC Public Health. 2014;14:891.

10. R. Laniado-Laborín SN-Á. Tuberculosis outbreak among health care workers in a general hospital. Rev Inst Nac Enfermedades Respir. 2007;20(3):189-94.

11. Costa JCT da, Silva R, Ferreira J, Nienhaus A. Active tuberculosis among health care workers in Portugal. J Bras Pneumol Publicaçäo Of Soc Bras Pneumol E Tisilogia. octubre de 2011;37(5):636-45.

12. Hosoglu S, Tanrikulu AC, Dagli C, Akalin S. Tuberculosis among health care workers in a short working period. Am J Infect Control. febrero de 2005;33(1):23-6.

13. Fica C A, Ramonda C P, Jemenao P MI, Zambrano G A, Cifuentes D M, Febré V N, et al. Tuberculosis in health care workers from a public health service in Santiago, Chile. Rev Chil
Infectol Órgano Of Soc Chil Infectol. febrero de 2009;26(1):34-8.

14. Organización Panamericana de la Salud. La tuberculosis en la Región de las Américas, Informe Regional 2013. Washington, DC. 2014. Disponible en http://www.paho.org/hq/ index.php?option $=$ com_docman $\&$ task=doc_view \&Itemid $=270 \&$ gi $\mathrm{d}=29808$ \&lang=es.

15. Alarcon Guizado Antonieta. Situación actual de la gestión en el control de TB en el Perú: análisis epidemiológico y operacional- Minsiterio de Salud [Internet]. 2015 sep [citado 12 de julio de 2015]; Lima- Perú. Recuperado a partir de: http://190.223.45.115/ newtb/Archivos/RecursoInformacion/20150930144300.pdf

16. Escombe AR, Huaroto L, Ticona E, Burgos M, Sanchez I, Carrasco L, et al. Tuberculosis transmission risk and infection control in a hospital emergency department in Lima, Peru. Int J Tuberc Lung Dis Off J Int Union Tuberc Lung Dis. septiembre de 2010;14(9):1120-6.

17. Alonso-Echanove J, Granich RM, Laszlo A, Chu G, Borja N, Blas R, et al. Occupational transmission of Mycobacterium tuberculosis to health care workers in a university hospital in Lima, Peru. Clin Infect Dis Off Publ Infect Dis Soc Am. 1 de septiembre de 2001;33(5):589-96.

18. Munayco CV. Prevalencia de infección por tuberculosis en trabajadores de salud. Red de Salud Bonilla-Callao. Informe técnico: Dirección General de Epidemiología- Ministerio de Salud. 2008.

19. Bonifacio N, Saito M, Gilman RH, Leung F, Cordova Chavez N, Chacaltana Huarcaya J, et al. High risk for tuberculosis in hospital physicians, Peru. Emerg Infect Dis. julio de 2002;8(7):747-8.

20. Hohmuth BA, Yamanija JC, Dayal AS, Nardell E, Salazar JJ, Smith Fawzi MC. Latent tuberculosis infection: risks to health care students at a hospital in
Lima, Peru. Int J Tuberc Lung Dis. 1 de octubre de 2006;10(10):1146-51.

21. Pérez-Lu JE, Cárcamo CP, García PJ, Bussalleu A, Bernabé-Ortiz A. Tuberculin skin test conversion among health sciences students: A retrospective cohort study. Tuberc Edinb Scotl. Marzo de 2013;93(2):25762.

22. Danilla M, Gave J, Martinez N. Tuberculosis ocupacional en un hospital general de Lima, Perú. Revista de la Sociedad Peruana de Neumología. 2005;49(2):101-3.

23. Accinelli Tanaka R, Noda Milla J, Bravo Padilla E, Galloso Benites M, López Oropeza L, Da Silva Caballero $\mathrm{J}$, et al. Enfermedad tuberculosa entre trabajadores de salud. Acta Médica Peru. Enero de 2009;26(1):35-47.

24. Nakandakari M, Rosa DD la, Gutierrez J, Bryson W. Tuberculosis en trabajadores de salud: Estudio epidemiológico y clínico en el Hospital Nacional Hipólito Unanue. Rev Medica Hered. 26 de septiembre de 2014;25(3):129.

25. Sanchez M, Marlene N. Características epidemiológicas de los trabajadores con diagnóstico de tuberculosis. Hospital Sergio E. Bernales, 2015. Univ. de San Martín Porres - USMP [Internet]. 2015 [citado 15 de abril de 2016]; Recuperado a partir de: http:// www.repositorioacademico.usmp.edu. pe/handle/usmp/1281

26. Alarcon Guizado Antonieta. Situación de la prevención y control de la tuberculosis en el Perú-Minsiterio de Salud [Internet]. 2012 sep [citado 12 de julio de 2015]; Lima, Perú. Recuperado a partir de: http://190.223.45.115/ newtb/Archivos/RecursoInformacion/20130419094051.pdf

\section{Correspondencia: Mirtha Gabriela Soto}

Cabezas

Dirección: Calle Daniel Olaechea 199. Lima 11, Perú. CDC-Ministerio de Salud. Lima, Perú. Teléfono: 6354500 anexo 129

Correoelectrónico:gsoto@dge.gob.pe 